\title{
The re-commissioning of a vent and reflux condensation research facility for vacuum and atmospheric operation
}

\author{
J. C. Sacramento Rivero \& P. J. Heggs \\ School of Chemical Engineering and Analytical Sciences, \\ The University of Manchester, UK
}

\begin{abstract}
The vent and reflux condensers are condensers where the primary mode of operation is condensation. Double-pipe vertical condensers are commonly used for their study. On the process side, a vapour mixture enters from the bottom of a vertical tube and as it flows upwards it condenses at the cooled tube wall, forming a falling film flowing counter-current to the vapour. On the other side of the tube, the coolant flows downwards, counter-current to the rising vapour but co-current to the falling condensate film. This paper discusses the re-commissioning of the vent/reflux condensation research facility in the Morton Laboratory at the University of Manchester. The experimental facility was encountered in an inoperable condition after the physical refurbishment of the Morton Laboratory. The sequence of re-commissioning actions taken is detailed and discussed, including instrumentation calibration, the revision and writing of Standard Operation Procedures and modifications to the P\&ID. Most of the instrumentation has been connected to the Emerson DeltaV ${ }^{\circledR}$ control system, allowing run-time data acquisition and automated control of the main process variables. A number of operational problems were encountered during the re-commissioning stages and the solution approach is described. The effect of the new and additional instruments/measurements on the mass and energy balances over the facility for previous and preliminary experimental investigations are detailed.
\end{abstract}

Keywords: vent condenser, reflux condenser, re-commissioning, instrumentation, control system. 


\section{Introduction}

The vent and reflux condensers are condensers where the primary mode of operation is condensation. Vent condensers achieve the highest possible condensation of the vapours, whereas reflux condensers only aim for a given separation, letting the most volatile component remain in the vapour phase. Therefore the difference between these two devices is just the operation conditions under which the same physical apparatus is run. Typical applications of reflux condensation are in overhead condensers of distillation columns, U-tube steam generators and two-phase closed loop thermosyphons. Vent condensers are often used to knock-out the vapour content from wet-air streams, such as those found in stirred-tank reactors or in the vent cooling section of aircooled steam condensers.

For the research of reflux/vent condenser mechanisms, double pipe heat exchangers are often used (see fig. 1). On the process side, a vapour mixture enters from the bottom of a vertical tube and as it flows upwards it condenses at the cooled tube wall, forming a falling film flowing counter-current to the vapour. On the other side of the tube, the coolant flows downwards, counter-current to the rising vapour but co-current to the falling condensate film. Hence, a reflux/vent condenser is a coupled system of 5 regions (see fig. 1): (1) the vapour flowing upwards, (2) the condensate film flowing downwards, (3) the heat transfer surface, (4) the coolant flowing downwards and (5) the control loop to provide the inlet temperature of the coolant.

The design information available for vent and reflux condensers presented in ESDU [1] normally leads to inadequate overdesign factors which can be as large as $70 \%$ or more, Jibb and Drogemuller [2]. This uncertainty in the design stage exists because the mechanisms of heat and mass transfer are not well known. Furthermore, the oversized condensers incur high capital and operational costs. Another concern in vent and reflux condensers operation is flooding. Flooding occurs when liquid is pushed upwards and exits along with the vapour at the top of the condenser.

The research facility was designed to study the coupled heat and mass transfer effects in the reflux condensation phenomena and allows the analysis of the main process variables using run-time data acquisition through the Emerson DeltaV ${ }^{\circledR}$ Distributed Control System (DCS). It also allows the experimenter to investigate the flooding conditions over a range of pressures up to atmospheric conditions. The main purpose of this research facility is to produce experimental data to support the development of design and performance evaluation methodologies which will reduce the large safety factors used so far in the design of this type of condenser and consequently reduce capital and operational costs, as well as energy consumption.

\section{Description of the research facility}

The research facility in the Morton Laboratory at the University of Manchester can be run in three different modes: reflux/vent condensation, single phase 
heating and single-phase cooling. Before starting a research programme it must be ensured that the proper configuration is adopted. The re-commissioning works were centred only on the reflux/vent condensation mode. The pipework connecting to irrelevant sections of the rig were duly isolated and tagged.

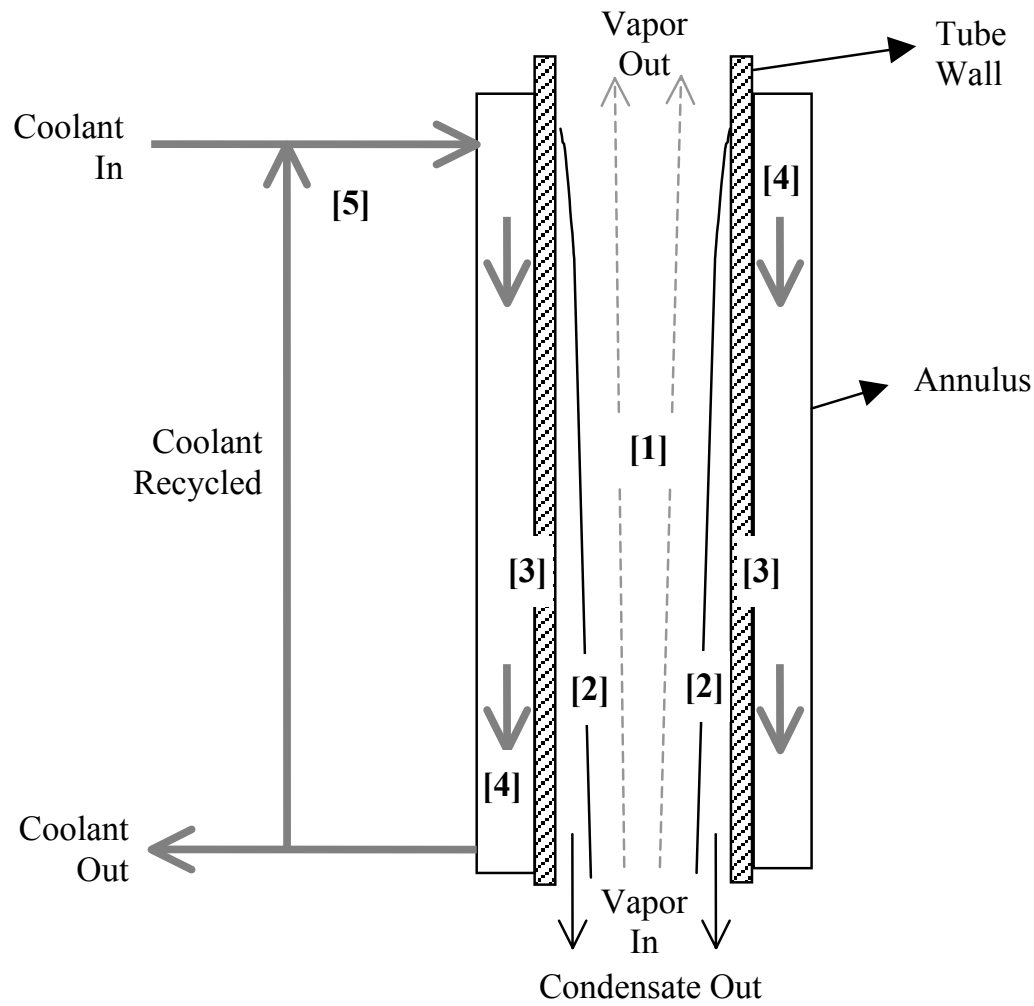

Figure 1: A sectional sketch of the vent and reflux condenser (not to scale) showing the 5 regions of interest.

The main section of the facility is the reflux/vent condenser or test section. This consists of a $3 \mathrm{~m}$ long vertical copper tube with an internal diameter of $0.028 \mathrm{~m}$. The annulus is a stainless steel jacket designed to allow the cooling water to enter in three different locations, so the overall length of reflux/vent condensation can be adjusted to $1 \mathrm{~m}, 2 \mathrm{~m}$ or $3 \mathrm{~m}$. Before the vapour mixture hits the test tube, it enters to an inlet pot that distributes evenly the vapour over the tube surface. The test tube is tapered at the bottom end in a $30^{\circ}$ angle. This should allow an increase of 5\% in vapour velocity before the system floods compared to the same tube without a taper (Palen and Yang [3]). Temperature measurements of the coolant are taken at the annulus inlet and outlet. Also, temperature measurements of the coolant and the tube wall are taken at 6 equally spaced points along the test tube length, allowing the validation of the local heat 
transfer coefficients. Furthermore, the heat flux profile on the coolant side can be evaluated.

The apparatus can operate using multicomponent mixtures and air. Fig. 2 shows a diagram of the rig. Initially, the selected mixture is fed to the boiling tank. Here, it is heated to its saturation temperature, which will vary with the pressure of the system. Normally, the rig is operated under vacuum, so boiling of the mixture is achieved at lower temperatures, although atmospheric operation is possible. The vacuum is generated by a two-stage Liquid Ring Pump (LRP) which uses water as the sealant liquid. The vapours generated in the boiling tank enter directly to the reflux condenser. There, the enthalpy of the rising vapours is removed by the cooling water flowing downwards in the annulus section. The cooling water flow can be manipulated to achieve partial or total condensation of the mixture. If used as a total condenser, all the vapours condense in the test section and are recycled to the boiling tank. If run as a partial condenser, the uncondensed vapours leaving at the top of the reflux condenser are driven to the after-condenser and knocked out. The two streams of condensate leaving both condensers are collected in the condensate collection pot and recycled by gravity to the boiling tank.

\subsection{The instrumentation}

The research facility is fully instrumented, covering all the variables required to close the mass and energy balances. The following paragraphs detail the different types of instrumentation currently in use.

\subsubsection{Temperature}

Most temperature readings are made using copper/constantan T-type thermocouples. These generate higher $\mathrm{mV}$ output per ${ }^{\circ} \mathrm{C}$ than other types of thermocouples in the operational range $\left(0-350^{\circ} \mathrm{C}\right)$. All the existing thermocouples needed to be re-calibrated since they were non-operational for a period of 3 years. The calibration procedure is described below.

Resistance Temperature Detectors (RTD) are used to measure the inlet and outlet temperatures in the oil heaters and the vapour temperature in the boiling tank. The output of the RTD model TC ${ }^{\circledR}$ Pt-100 is a 4-20 mA analog signal proportional to the sensed temperature. The RTD has a longer term stability but a slower response than the thermocouples. Despite the robustness of these meters, a linearity response check was made.

\subsubsection{Pressure}

Where visual pressure readings are desirable, normal manometers are placed, with operational ranges between 0 to 2 bar. These field readings are only to support the online values recorded through the DCS using Pressure Transmitters (PT). The PT consists of a membrane that distorts when pressure/vacuum is applied. This distortion generates a current (4-20 mA) proportional to the applied pressure. The models of the PT used in this facility are DRUCK ${ }^{\circledR}$ PT600 and PT500 with operational ranges varying within 0-1.6 bar, to allow the operation of the rig under vacuum and just above atmosphere. 


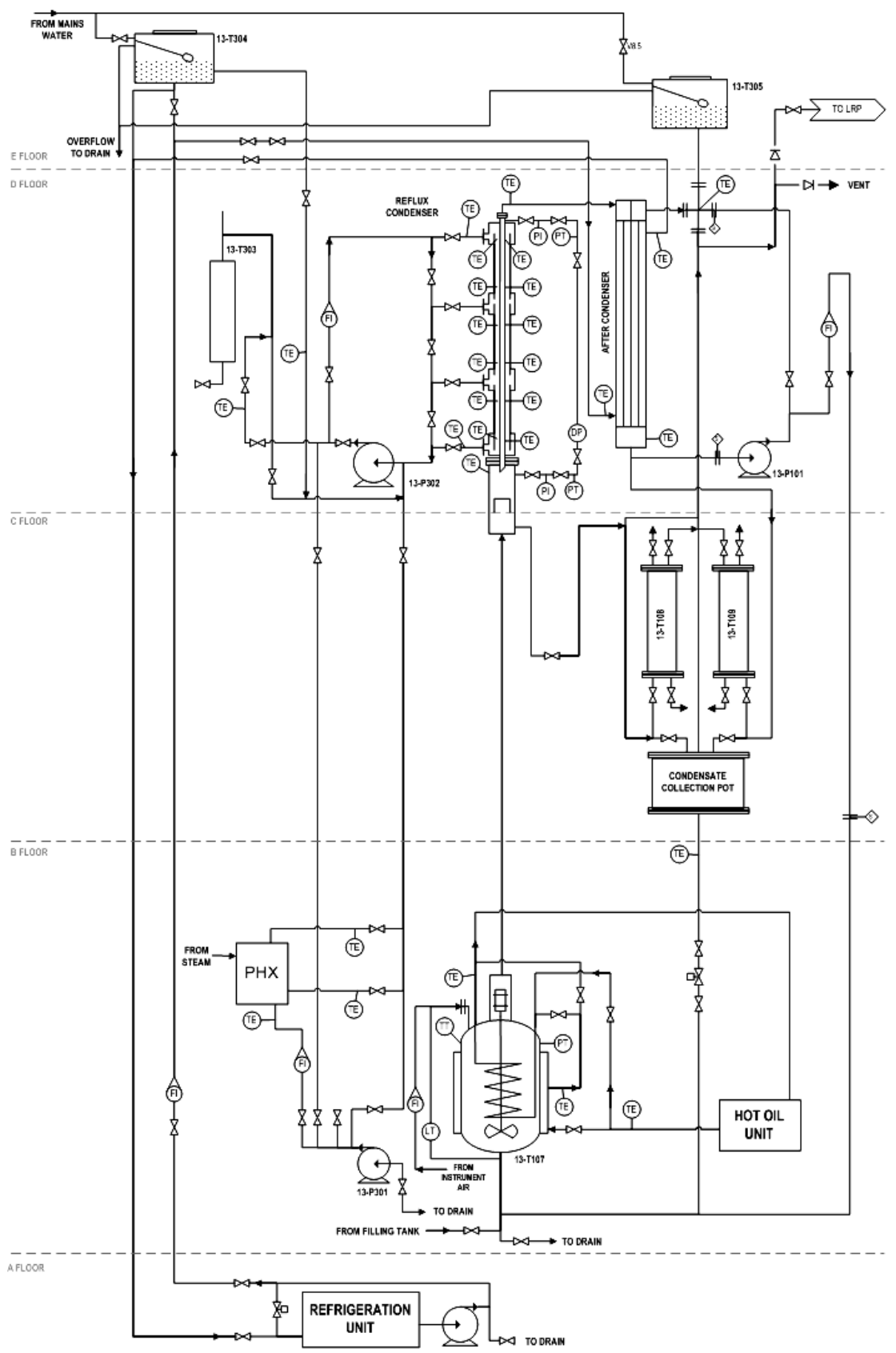

Figure 2: A diagram of the heat transfer research facility showing the main instrumentation. 
To measure the pressure drop across the test section, a Differential Pressure (DP) cell model Rosemount ${ }^{\circledR} 1151-\mathrm{E}$ is used to double-check the readings retrieved by two PTs located at the bottom and at the top of the test section. It has been observed that the DP cell reading is more accurate than that of the difference of the PTs readings.

\subsubsection{Flows}

All cooling water flows are measured with rotameters. These provide a visual reading of the volumetric flow. Since the measured fluid is water no further calibration of the sight glass scale was needed and the supplier calibration curves can be used. When the measured fluid is air, a correction for pressure is required so a pressure gauge was fitted at the air inlet of the rotameter. For the gravity make up in the test section cooling water loop, a scaled glass vessel (13-T303 in fig. 2) is used to manually read the volumetric flow.

For flow measurement of the process condensate, which vary in composition during normal operation, it was necessary to account for density changes. Coriolis flow meters provide not only accurate readings for small mass flow but also density measurements, which can be translated into composition estimates when the condensate is a multicomponent mixture.

An orifice plate is used to measure the hot oil flow. The differential pressure across the orifice plate is measured by a DP cell similar to that used to measure the pressure drop across the test section. The equation and algorithm used to estimate the flow from the measured differential pressure was taken from the relevant British Standards $[4,5]$ and it is of the form:

$$
\dot{V}=C_{d} A_{o} \sqrt{\frac{2 \rho \Delta P}{1-\beta^{4}}}
$$

where, $\dot{V}$ is the volumetric flow, $C_{d}$ is the coefficient of discharge, $A_{o}$ is the sectional area of the orifice, $\rho$ is the density of the fluid, $\beta$ is the orifice to pipe diameters ratio and $\Delta P$ is the measured differential pressure.

\subsection{The Distributed Control System (DCS)}

Most of the instrumentation is administrated by the Emerson DCS DeltaV ${ }^{\circledR}$. It allows continuous recording of compatible instrumentation and manipulating controllers. Every plant instrument sends a discrete or analog signal (current or voltage) and it is received by an $\mathrm{I} / \mathrm{O}$ card. The signal is converted to digital signal and sent to a Controller, within which all data will be processed and actions will be taken, in the case control valves. Both $\mathrm{I} / \mathrm{O}$ cards and controllers are inside Local Control Panels (LCP), located in the plant area close to the equipment. All signals received by the controllers are sent to operator stations within the Morton Laboratory control room. From there, they can be monitored and controlled using a graphical interface. 


\section{The re-commissioning stages}

The experimental facility was in an inoperable condition after the physical refurbishment of the Morton Laboratory. The LRP was physically displaced, almost all the insulation was torn up and several flanges were loose. The schedule of the re-commissioning works comprised the following stages:

1. An integrity check of the experimental apparatus.

2. Updating the documentation of the research facility.

3. The set up of the instrumentation.

4. The set up of the control system.

5. The insulation of the apparatus.

6. The uncertainty analysis.

\subsection{Checking the integrity of the experimental apparatus}

The first activity of the re-commissioning was a full pipe tracing, comparing the physical apparatus with the Process and Instrumentation Diagram (P\&ID). Several corrections had to be made to the P\&ID since various modifications were made during the refurbishment of the Morton Laboratory, i.e. additional valves and instrumentation. During the pipe tracing all the flanges and valves were checked so any loose bolts and blocked pipes were identified. The sections that were not relevant to the reflux/vent condensation experiments were isolated and the isolation valves were tagged to prevent accidental opening.

Once the line tracing was complete a hydraulic test was carried out in the cooling water side. Filling the cooling water system revealed several leaks and pertinent actions were taken. Finally, a "drop test" was made to first locate the air leaks locations and then quantify the residual air leakage. The LRP was used to generate vacuum $(0.1$ bar $)$ in the rig and a careful inspection of all flanges in the rig revealed the air leaks location. After tightening all the guilty flanges, the drop test was carried out. The test consists in putting vacuum into the system, closing the vacuum pump line and recording the time, $t$, that the system takes to reach a certain higher pressure. If the system volume, $V$, is known, the ideal-gas law is used to calculate the air mass flow, $\dot{M}$ as follows:

$$
\dot{M}=\frac{29}{t} \frac{V \Delta P}{R T}
$$

where, $\Delta P$ is the pressure difference along the test, 29 is the molecular mass of air and $T$ is the system temperature. Eqn. (2) assumes that air leaks at sonic velocity, i.e. at a constant rate over the whole test. Therefore, both initial and final pressures should be less than approximately 0.53 times atmospheric pressure (Ryans and Roper [6]). The air leakage was estimated at $0.12 \mathrm{~kg} / \mathrm{h}$ and it was considered acceptable so no further leak tests were made.

\subsection{The set up of the instrumentation}

In the case of new instrumentation, a selection procedure is needed to choose the most appropriate instrument type for the application. In the case of the 
thermocouples, the nature of the process, the operation range and the signal quality were the most important parameters, as stated above. Special attention was given to the selection of the coriolis meters. The occasional presence of two-phase flow in the condensate lines made it difficult to size the meter and most vendors warn about high instability of the output signal when two-phase flow is present. The recommended transmitter was the Foxboro ${ }^{\circledR}$ CFT50 which can cope with this situation. The meter was also slightly oversized in such a way that the presence of the gaseous phase will not cause the mass flow to exceed the meter range.

Once the selection is made, the installation of the instrument follows. If the instrument is to be connected to the DCS, the signal wire is selected and run from the terminal side of the instrument itself to the LCP and attached to its I/O card. When the DCS shows some reading from the instrument, it is ready for in situ calibration.

The calibration of any instrument consists in adjusting the output signal of the instrument to the desired range. For example for a DP cell, a known pressure is applied to the high-pressure terminal (leaving the low pressure terminal open to atmosphere) and the known differential pressure is compared to the reading retrieved from the DCS. If the values do not match, the pertinent corrections to the Zero and Span values in the DP cell are made and the test is repeated several times until the repeatability of the readings is acceptable. This procedure applies to all instrumentation with linear output, such as PTs, RTDs and Coriolis meters.

The thermocouples output is not linear, so a different approach is required. The calibration of the thermocouples is made using a Signal Characterisation Block (SCB) in the instrument configuration in the DCS. The SCB stores a comparison table between the recorded value of each thermocouple and a reference value considered to be accurate. In this case the reference temperature was taken from a Platinum Resistance Thermometer (PRT) which was calibrated by the vendor to an accuracy of $\pm 0.03^{\circ} \mathrm{C}$ for the used range. The readings of the PRT showed to be in good agreement with an alcohol thermometer as well.

Each thermocouple was exposed to different steady temperatures within the range of the process conditions and it was compared against the PRT reading. The results were averaged and these calibration parameters were stored in a SCB for each thermocouple. During normal operation the DCS will interpolate the value the PRT would have using the thermocouple sensed value and the calibration parameters.

\subsection{The set up of the control system}

After all the instrumentation is properly connected to their respective LCP, each instrument needs to be configured in the DCS. Care must be taken to (1) ensure each reading in the DCS front end shows the correct instrument signal, (2) check the read scale in the DCS configuration matches the calibrated scale of the instrument and (3) check that the calibration parameters are correctly input in the $\mathrm{SCB}$ in the case of instruments with non-linear output.

It was also necessary to re-draw the front end graphics to accommodate for the changes in the P\&ID, that is new pipework and additional instrumentation. 


\subsection{Updating the documentation of the research facility}

After all modifications were made, the facility P\&ID was corrected and updated. The equipment and valves were re-numbered to conform to the general Morton Laboratory numbering system. New Standard Operation Procedures (SOP) were written for the calibration of the instrumentation and the existing ones were updated to conform to the new configuration of the facility and the university's new safety policy.

Before any physical change was made to the rig, a Management of Change (MOC) form had to be submitted, explaining the nature of the job and the key safety issues that may arise from the modification, as well as the impact the new addition may have in the normal operation of the apparatus. Safety documentation also includes Risk Assessments (RA) and COSHH forms, since some research using water/methanol mixtures is programmed. Finally, a full HAZOP study is programmed to evaluate the hazards the operation of the rig may involve.

\subsection{The insulation of the apparatus}

The insulation thickness of the pipework was calculated in such a way the external temperature do not exceed $50^{\circ} \mathrm{C}$ for safety purposes. The test section was insulated to minimise the heat losses as those temperature measurements are critical for the condenser performance evaluation. The calculation of the insulation thickness contemplated the following resistances: the forced convection inside the pipe, the pipe wall resistance, the insulation material resistance, the external radiation to the ambient and the external free convection.

\subsection{The uncertainty analysis}

All the acquired data have an embedded uncertainty that comes from the nature of the instrument used. When correlations are used to calculate certain parameters an additional uncertainty adds to the final result. To evaluate the overall uncertainty in the presented results the following expression must be used:

$$
\frac{\delta R}{R}=\left[\left(a \frac{\delta X_{1}}{X_{1}}\right)^{2}+\left(b \frac{\delta X_{2}}{X_{2}}\right)^{2}+\left(c \frac{\delta X_{3}}{X_{3}}\right)^{2}+\cdots+\left(N \frac{\delta X_{n}}{X_{n}}\right)^{2}\right]^{1 / 2}
$$

where $\delta X_{i}$ is the uncertainty of the measured variable $X_{i}$ expressed as a percentage. Eqn. (3) assumes that the result $R$ is a variable that can be expressed in a pure product form, i.e. $R=X_{1}^{a} X_{2}^{b} X_{3}^{c} \cdots X_{n}^{N}$.

Moffat [7] describes three types of uncertainty analysis: zeroth-, first and nth-order uncertainties. Following Moffat's nomenclature, the results presented here correspond to the first order uncertainty for single sample experiments. This is intended to describe the scatter that should be expected in a set of observations using the given apparatus and measuring system. This estimate includes all sorts of variable errors, but does not include fixed errors of any kind. It is reported to 
compare set of data of experiments done in the same apparatus with the same instrumentation, but should not be used to compare experiment results from different rigs, as the fixed errors are not accounted for. The first order uncertainty is obtained using the chi-squared statistic applied to the data retrieved from an auxiliary experiment. From this analysis it can be expected a scatter of the results in the mass and energy balances between 3 and $6 \%$.

\section{Future work}

With the research facility as it is now, the researcher work splits between the manual data acquisition of some instruments and processing the data of all instrumentation using spreadsheets. A great time saving for the researcher would be to implement a real-time data analysis in Delta ${ }^{\circledR}$ in such a way that the mass and energy balances were calculated as the instrument data are read. It will also permit the identification of steady-state operation of the system based on the energy balances rather than in just isolated values of some variables. To achieve this, it would only require writing the same equations in the spreadsheet in Calculator modules within DeltaV ${ }^{\mathbb{B}}$. However and obviously all rotameters and visual instruments have to be substituted by instrumentation with an output signal, which is expensive.

If all the instrumentation in the rig is connected to the DCS it would also be possible to include the data uncertainty analysis in run-time, as explained by Moffat [7]. This would allow identifying which measurements are the major source of error during certain experimental run and would prevent the experimenter of doing potential unusable runs.

\section{References}

[1] ESDU, Engineering Sciences Data Unit (Data Item 89038). Reflux Condensation in Vertical Tubes, 1989.

[2] Jibb, R.J. \& Drogemuller, P., Design and Application of Reflux Condensers for Separating Vapour Mixtures. $3^{\text {rd }}$ International Conference on Process Intensification, 1999.

[3] Palen, J. \& Yang, Z.H., Reflux Condensation Flooding Prediction: Review of Current Status, Chemical Engineering Research and Design. Trans IChemE, 79 Part A, pp. 463-469, 2001.

[4] British-Standard. Measurement of fluid flow by means of differential pressure devices, BSi. BS1042-1.1:1992 renumbered BS EN ISO 51671:1997, 1997.

[5] British-Standard. Measurement of fluid flow in closed conduits, BSi. BS1042-1.4:1998, 1998.

[6] Ryans, J.L. \& Roper, D.L., Process Vacuum System Design \& Operation, ed. McGraw-Hill, 1986.

[7] Moffat, R.J., Describing the Uncertainties in Experimental Results. Experimental Thermal and Fluid Science, 1, pp. 3-17, 1988. 